Ann. Biol. anim. Bioch. Biophys., I976, 16 (5), 687-697.

\title{
VARIATIONS DU COMPORTEMENT ALIMENTAIRE, DE LA CROISSANCE ET DE LA DIGESTIBILITÉ CHEZ DES LAPINS A CANAL PANCRÉATIQUE LIGATURÉ
}

\author{
Jacqueline CATALA \\ Institul de Physiologie, \\ Laboratoire de Physiologie de la Digestion et de la Nutrition \\ 2 rue François-Magendie, \\ 31400 Toulouse
}

RÉSUMÉ

\begin{abstract}
A fin d'étudier l'importance de la digestion pancréatique chez le Lapin, nous avons pratiqué des ligatures du canal de Wirsung et nous avons étudié, chez ces animaux, le comportement alimen- taire, la croissance et le coefficient d'utilisation digestive des matières azotées.

$\mathrm{Au}$ cours de notre expérimentation, nous avons utilisé 30 lapins nourris aux aliments secs du commerce à $\mathrm{I} 6 \mathrm{p}$. roo de protéines.

Une première expérimentation de ro semaines concernant la prise alimentaire et la croissance, a porté sur un lot de $I 6$ lapins : 8 témoins $T$ et 8 à canal pancréatique ligaturé $P$. Il apparaît une diminution de la prise de nourriture jusque vers la $4^{\mathrm{e}}$ semaine post-opératoire chez les T et la $6^{e}$ semaine chez les $\mathrm{P}$. Chez ces derniers, on observe, parallèlement à cette période, un ralentissement de croissance, mais au-delà l'évolution pondérale de ces animaux reprend normalement.

Une deuxième expérimentation sur le coefficient d'utilisation digestive des matières azotées, qui a concerné 2 lots de 7 animaux $T$ et $P$, nous a permis d'évaluer une diminution de la digestion protéique de $30 \mathrm{p}$. Ioo environ, 4 semaines après la pose de la ligature.
\end{abstract}

\section{INTRODUCTION}

Depuis plus d'un siècle, de nombreux auteurs se sont intéressés à la fonction exocrine du pancréas chez différentes espèces, mais il est étonnant de constater que peu de travaux concernent le Lapin (BAXTER, I93 I RothMAN, I974; CoRRING et al., I972). Les recherches effectuées chez cet animal sur le pancréas, s'adressent généralement à la structure glandulaire (TISCORNIA et DREILING, I966) délaissant tout ce qui concerne la digestion et le comportement. A notre connaissance, seuls BAcQuEs et al. (I970) et Demigne et al. (I974) ont fait des études dans ce sens. 
Ce manque d'intérêt pour le Lapin résulte, peut-être, de la complexité de ses processus digestifs ( $\mathrm{L}_{\mathrm{F}} \mathrm{BAS}, \mathrm{I}$ 669) due en grande partie à la présence des microorganismes dans les différentes poches digestives.

Il suffit de rappeler qu'au niveau de l'estomac, VIALLARD (I968) a montré qu'une partie de la flore réingérée est détruite par un agent bactériolytique, différent de la pepsine, tandis qu'une autre partie de cette flore prolifère à $\mathrm{pH}$ acide en ce même lieu.

Nous avons également pu mettre en évidence qu'il existe une prolifération importante de microorganismes tout le long de l'intestin grêle malgré la sécrétion de sucs digestifs intestinaux et pancréatiques (CATALA et Bonnafous, I974).

C'est au niveau cæcal que semble se situer le métabolisme microbien le plus intense. Cependant, BonNafous (I968) a montré que le côlon est un organe également très actif. Cet auteur a pu isoler un agent bactériolytique puissant, capable de lyser au cours du transit colique 7 I p. Ioo des microorganismes.

Dans de telles conditions, nous nous sommes demandés si le rôle des sécrétions digestives, classiquement connu dans les parties antérieures de l'intestin grêle, gardait toute sa signification. Le but de ce travail tend à préciser le rôle de la sécrétion pancréatique exocrine chez le Lapin.

Les travaux effectués dans ce sens chez d'autres espèces, telles que le Chien (Svecova, I964), le Poulet (Lepkowsky et al., I974) le Porc (ANDERson et Ash, I97I ; PEKAs et al., I964) et le Rat (Clowes et McPHERSON, I95I), signalent que la suppression de la sécrétion pancréatique entraîne des désordres digestifs qui s'accompagnent souvent, chez ces animaux, d'une perte de poids plus ou moins importante. Il devient alors nécessaire d'apporter des modifications au régime alimentaire pour maintenir les animaux dans de bonnes conditions expérimentales.

Pour aborder cette étude, après suppression de l'arrivée du suc pancréatique par ligature du canal de Wirsung (CATALA, I972), nous avons recherché, dans un premier temps, le comportement de nos animaux et plus particulièrement, leur alimentation et leur croissance; dans un deuxième temps, nous avons fait une étude sur la digestibilité protéique (coefficient d'utilisation digestive de la matière azotée : $\mathrm{CUD}_{a}$ ).

\section{TECHNIQUES}

Cette étude a été conduite en automne et porte sur 30 lapins de race commune, âgés de $\mathrm{I}_{4}$ à I 5 semaines, d'un poids moyen de $2,5 \mathrm{~kg}$. Les expériences sont réalisées pendant la deuxième étape de la phase de croissance (PRUD'HON, I967). Les animaux sont nourris pendant I mois, ad libitum, aux aliments secs du commerce dont la composition est la suivante : p. roo matière cellulosique I4, matière minérale $\mathrm{I}_{0}$, humidité $\mathrm{I} 4$, matières protéiques brutes $\mathrm{I} 6$, matière grasse 2 et vitamines $\mathrm{A}$, $\mathrm{D}_{3}, \mathrm{~B}_{2}, \mathrm{~B}_{6}, \mathrm{~B}_{12}, \mathrm{~K}, \mathrm{PP}$, choline et acide panthoténique.

\section{Consommation alimentaive et croissance}

Les animaux sont divisés en 2 lots : $\mathrm{I}$ lot de 8 animaux témoins $\mathrm{T}$ sur lesquels nous pratiquons une opération à blanc (anesthésie et laparotomie); 1 lot de 8 animaux $P$ dont nous ligaturons le canal de Wirsung (CATALA, 1972).

A partir de ce jour, et pendant ro semaines, les animaux sont replacés dans leur cage individuelle respective. Les pesées des animaux et les pesées de nourriture distribuée, gaspillée et refusée, sont effectuées quotidiennement. Nous avons regroupé, dans nos tableaux, toutes ces valeuts en valeurs hebdomadaires. 


\section{Coefficient d'utilisation digestive des protéines}

L'étude du coefficient d'utilisation digestive des protéines est faite sur I4 lapins divisés en 2 lots : 7 lapins subissent l'opération à blanc et 7 autres la ligature du canal pancréatique.

Deux périodes de collectes ont été réalisées sur chacun des deux groupes; avant les opérations et 4 semaines après.

Les animaux sont placés dans des cages à métabolisme individuelles pour une période de Io jours Ils reçoivent de l'eau ad libitum et sont libres de pratiquer la cæcotrophie. La ration alimentaire de $200 \mathrm{~g}$ est distribuée tous les matins, les pesées de la nourriture résiduelle se font au même moment. Les fèces de chaque lapin sont recueillies. pesées à l'état frais et placées à l'étuve à $90^{\circ} \mathrm{C}$ pendant 48 heures, puis repesées à l'état sec et enfin finement broyées. lyser".

Les dosages d'azote sont effectués à partir de $20 \mathrm{mg}$ de poudre au "Coleman Nitrogen Ana-

Remarque : par suite d'avaries et de difficultés de récupération totale des urines, nous ne pouvons rapporter ici que les valeurs concernant le CUD.

La formule utilisée correspond au coefficient d'utilisation digestive apparent des protéines :

$$
\frac{\mathrm{N} \text { ingéré }-\mathrm{N} \text { fécal }}{\mathrm{N} \text { ingéré }} \times \text { ıоo }=\mathrm{CUD}_{a}
$$

\section{RÉSULTATS}

\section{I. - Prise de nourriture}

De ces premiers résultats, regroupés dans le tableau $I$, sur la prise de nourriture hebdomadaire entre les $T$ et les $P$, pour une période post-opératoire de ro semaines, il ressort que la quantité de granulés ingérés par les deux types d'animaux n'est pas significativement différente $\left(\mathrm{F}_{158}^{1}=0,50\right)$.

\section{TABLEAU I}

Prise de nourriture hebdomadaire des lapins $\mathrm{T}$ et $\mathrm{P}$ au cours des 10 semaines d'expérience

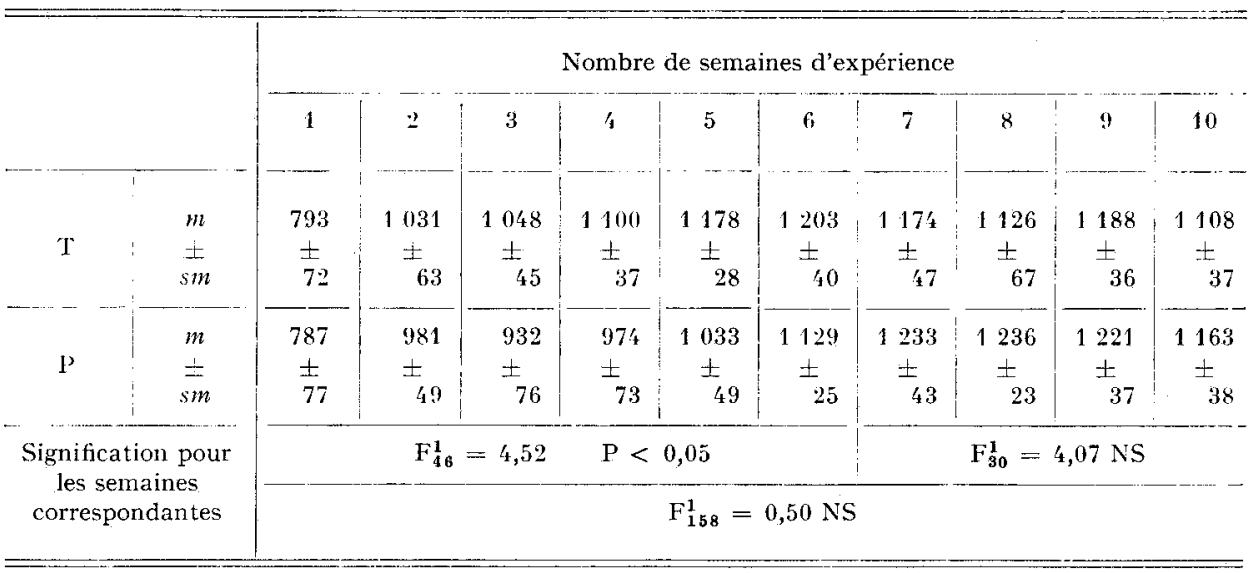


Cependant, il apparait que les P consomment moins de nourriture que les T les 6 premières semaines et systématiquement plus les 4 semaines suivantes. Or, l'analyse de variance effectuée sur les 6 premières semaines montre bien que les $P$ ingèrent moins d'aliments que les $\mathrm{T}, \mathrm{F}_{46}^{1}=4,52(\mathrm{P}<0,05)$. Par contre, les 4 semaines suivantes nous n'avons pas trouvé de différence significative $\mathrm{F}_{30}^{1}=4,07$. L'estimation de ce surplus d'alimentation est, en effet, très faible puisqu'il ne représente que $8 \mathrm{~g}$ par jour.

I1 faut noter que la première semaine qui suit l'opération, la consommation alimentaire des deux types d'animaux est identique et ne représente que $70 \mathrm{p}$. Ioo environ de leur ingestion moyenne habituelle ( $\mathrm{I}$ IOO g). Par la suite, la reprise de la consommation normale de granulés se fait de façon plus rapide chez les $T$ que chez les $P$ puisqu'elle se produit respectivement à 4 et 6 semaines après les opérations.

\section{2. - Croissance des animaux}

I'analyse de variance effectuée sur les poids hebdomadaires des animaux $\mathrm{T}$ et $\mathrm{P}$ durant les Io semaines expérimentales donne un $F_{158}^{1}=\mathbf{1 2} 2,7^{2}$, hautement significatif (tabl, 2).

TABLEAU 2

Poids hebdomadaives des $\mathbf{T}$ et $\mathbf{P}$ durant les 10 semaines d'expérience

\begin{tabular}{|c|c|c|c|c|c|c|c|c|c|c|c|c|}
\hline & & \multicolumn{11}{|c|}{ Nombre de semaines d'expérience } \\
\hline & & 0 & 1 & $\stackrel{2}{2}$ & 3 & $t_{t}$ & 5 & 6 & $\tau$ & 8 & 9 & 10) \\
\hline $\mathrm{T}$ & $\begin{array}{c}m \\
\pm \\
s m\end{array}$ & $\begin{array}{c}2,591 \\
\underset{0,047}{ \pm}\end{array}$ & $\begin{array}{c}2,492 \\
\pm \\
0,047\end{array}$ & $\begin{array}{c}2,666 \\
\pm \\
0,067\end{array}$ & $\begin{array}{c}\cdot, 779 \\
\stackrel{+}{=} \\
0,065\end{array}$ & $\begin{array}{c}2,871 \\
\pm \\
0,055\end{array}$ & $\begin{array}{c}3,009 \\
\pm \\
0,076\end{array}$ & $\begin{array}{c}3,120 \\
\underset{0,076}{ \pm}\end{array}$ & $\begin{array}{c}3,219 \\
\pm \\
0,073\end{array}$ & $\begin{array}{c}3,290 \\
\pm \\
0,077\end{array}$ & $\begin{array}{c}3,405 \\
\pm \\
0,070\end{array}$ & $\begin{array}{c}3,470 \\
\pm \\
0,074\end{array}$ \\
\hline $\mathrm{P}$ & $\begin{array}{c}m \\
\pm \\
s m\end{array}$ & $\begin{array}{c}2,543 \\
\pm \\
0,064\end{array}$ & $\begin{array}{c}2,511 \\
\pm \\
0,063\end{array}$ & $\begin{array}{c}2,567 \\
\pm \\
0,07\end{array}$ & $\begin{array}{c}2,655 \\
\pm \\
0,059\end{array}$ & $\begin{array}{c}2,675 \\
\pm \\
0,055\end{array}$ & $\begin{array}{c}2,783 \\
\pm \\
0,056\end{array}$ & $\begin{array}{c}2,850 \\
\pm \\
0,054\end{array}$ & $\begin{array}{c}2,980 \\
\pm \\
0,048\end{array}$ & $\begin{array}{c}3,055 \\
\pm \\
0,053\end{array}$ & $\begin{array}{c}3,131 \\
\stackrel{+}{\overrightarrow{7}} \\
0,067\end{array}$ & $\begin{array}{c}3,171 \\
\pm \\
0,076\end{array}$ \\
\hline \multicolumn{2}{|c|}{ Signification } & \multicolumn{6}{|c|}{$F_{155}^{1}=12,72$} & \multicolumn{5}{|c|}{$P<0,001$} \\
\hline
\end{tabular}

Au terme de la période expérimentale, la différence de poids observée entre les deux types d'animaux peut être évaluée à environ $9 \mathrm{p}$. Ioo si on la rapporte au poids total de l'animal ou à $28 \mathrm{p}$. roo si on la rapporte au gain de poids durant les ro semaines expérimentales.

Il semble que ce ralentissement de croissance qui apparaît chez les $P$ ne se fasse pas de façon régulière alors que les deux groupes d'animaux $T$ et $P$ étaient homogènes au temps initial de l'expérimentation.

En effet, on observe, la première semaine suivant l'opération, une chute pondérale de tous les animaux mais avec une perte de poids trois fois supérieure chez les $T$ que chez les animaux $\mathrm{P}(99 \mathrm{~g}$ contre $32 \mathrm{~g}$ ).

Par ailleurs, nous avons établi les droites de régression à partir des poids hebdo- 


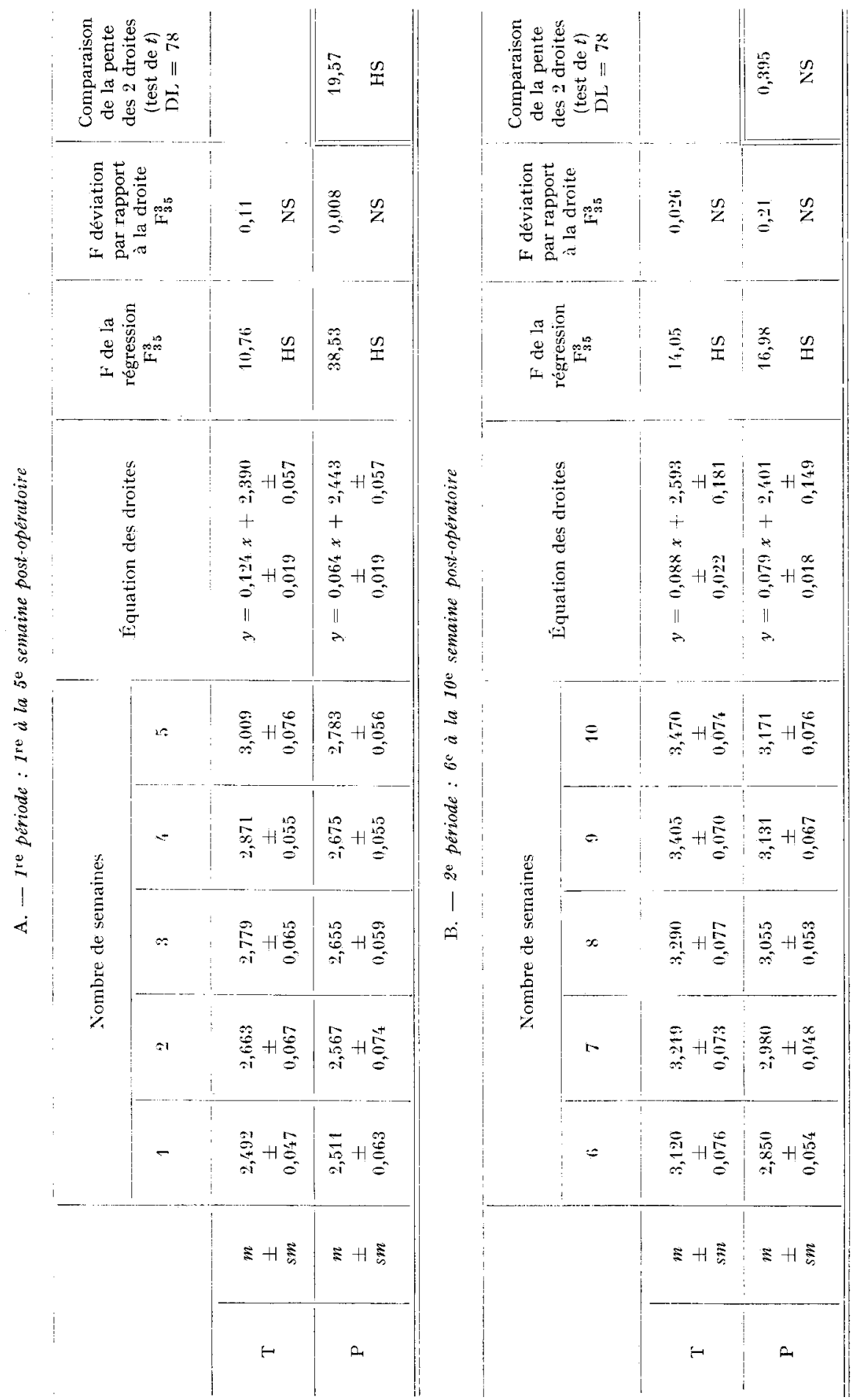




\section{TABLEAU 4}

$\mathrm{CUD}_{a}$ des animaux $\mathrm{T}$ avant et 4 semaines après l'opération fictive

\begin{tabular}{|c|c|c|c|c|c|c|c|c|c|}
\hline & \multicolumn{7}{|c|}{ Nombre d'animaux } & \multicolumn{2}{|c|}{$!$} \\
\hline & 1 & 2 & 3 & 4 & 5 & 6 & 7 & & \\
\hline $\mathrm{T}$ & $\quad 65,9$ & 62,3 & 79,0 & 60,8 & 70,7 & 66,6 & 71,6 & $68,12 \pm 2,34$ & $t=0,6^{\prime} \mathrm{t}$ \\
\hline $\mathrm{T}_{4}$ & 60,1 & 65,1 & 72,4 & 65,3 & 64,6 & 70 & 67,2 & $66,32 \pm 1,51$ & NS \\
\hline
\end{tabular}

$\mathrm{T}_{4}$ : animaux $\mathrm{T} 4$ semaines après l'opération fictive.

\section{TABLEAU 5}

$\mathrm{CUD}_{a}$ des animaux $\mathrm{P}$ avant et 4 semaines après l'opération

\begin{tabular}{|c|c|c|c|c|c|c|c|c|c|}
\hline & \multicolumn{7}{|c|}{ Nombre d'animaux } & \multirow{2}{*}{$m \pm s m$} & \multirow{2}{*}{ Signification } \\
\hline & 1 & 2 & 3 & 4 & 5 & 6 & 7 & & \\
\hline $\mathrm{P}$ & 63,9 & 64,5 & 69,0 & 68,5 & 71,1 & 60,9 & 59 & $65,27 \pm 1,68$ & $t=6,96$ \\
\hline$P_{4}$ & 37,3 & 47,3 & $46,,^{\prime}$ & 1,7 & $2 / 4,2$ & 46,7 & 42,1 & ${ }^{\prime}(0,81 \pm 3,08$ & $p<0,001$ \\
\hline
\end{tabular}

$P_{1}$ : animaux $P_{4}$ semaines après l'opération.

\section{TABLEAU 6}

CUD théorique des substances non azotées chez les $\mathrm{T}$ et $\mathrm{P} 4$ semaines après les opérations

\begin{tabular}{|c|c|c|c|c|c|c|c|c|c|}
\hline & \multicolumn{7}{|c|}{ Nombre d'animaux } & \multirow{2}{*}{$m \pm s m$} & \multirow{2}{*}{ Signification } \\
\hline & 1 & 2 & 3 & 4 & 5 & 6 & 7 & & \\
\hline $\mathrm{T}$ & 68,2 & 68,4 & 73,4 & 66,7 & 75,3 & 66,7 & 65,0 & $69,10 \pm 1,43$ & $t=2,37$ \\
\hline $\mathrm{P}$ & 53,2 & 69,1 & 69,4 & 60,8 & 65,3 & 62,1 & 61,3 & $63,02 \pm 2,11$ & $p<0,05$ \\
\hline
\end{tabular}


madaires des $\mathrm{T}$ et des $\mathrm{P}$ pour les deux périodes de l'expérimentation, situées de la $\mathrm{I}^{\mathrm{re}}$ à $\mathrm{la} 5^{\mathrm{e}}$ semaine, et de la $6^{\mathrm{e}}$ à la Io $^{\mathrm{e}}$ semaine post-opératoire.

Des résultats consignés dans le tableau 3 , il apparaît pour la $I^{r e}$ période, aussi bien chez les $T$ que chez les $\mathrm{P}$, une courbe de régression linéaire ( $\mathrm{T}: \mathrm{F}_{35}^{1}=10,76 \mathrm{HS}$; $\mathrm{F}_{35}^{3}=0$, I I NS) $\left(\mathrm{P}: \mathrm{F}_{35}^{1}=38,53 \mathrm{HS} ; \mathrm{F}_{35}^{3}=0,008 \mathrm{NS}\right)$ dont les pentes sont différentes $(t=\mathrm{I} 9,57 \mathrm{HS})$.

Par contre, pour la $2^{\mathrm{e}}$ période, nous observons toujours chez les deux types d'animaux $T$ et $P$, une courbe de régression linéaire $\left(T: F_{35}^{1}=I_{4}, 05 \mathrm{HS} ; \mathrm{F}_{35}^{3}=0,026\right.$ NS) $\left(\mathrm{P}: \mathrm{F}_{35}^{1}=\mathrm{I} 6,98 \mathrm{HS} ; \mathrm{F}_{35}^{3}=0,2 \mathrm{I} \mathrm{NS}\right)$, dont, cette fois, les pentes sont identiques $(t=0,395 \mathrm{NS})$.

Ces résultats montrent donc que la ligature du canal pancréatique entraîne bien un ralentissement de croissance les 5 premières semaines post-opératoires et que, par la suite, la prise de poids devient identique pour les deux lots d'animaux.

\section{3. - Coefficient d'utilisation digestive des substances azotées}

Ces résultats montrent que le CUD azoté moyen de nos animaux adultes témoins (tabl. 4) qui est de 68,12 $\pm 2,34$ points ne s'abaisse que très faiblement puisque 4 semaines après il est encore de $66,32 \pm \mathrm{I}, 5 \mathrm{I}$ points : cette différence n'étant d'ailleurs pas significative $t=0,64 \mathrm{NS}$. Par ailleurs, toute diminution importante du CUDa chez les $\mathbf{P}$ ne sera donc pas imputable à une évolution spontanée de ce CUD ${ }_{a}$.

Chez les animaux $\mathrm{P}$ le $\mathrm{CUD}_{a}$ s'abaisse fortement puisqu'il passe de $65,27 \pm \mathrm{I}, 68$ à $40,8 \mathrm{I} \pm 3,08$ points, 4 semaines après la pose de la ligature, soit 24,46 points en moins (tabl. 5). On peut donc dire que la digestibilité semble diminuer de $30 \mathrm{p}$. Ioo environ.

A partir de ces données sur la digestibilité des substances protéiques, il nous a paru intéressant d'extrapoler des données théoriques concernant le CUD des substances " non azotées " qui pourraît être assimilé au CUD des substances "glucidiques ", si on estime que les $2 \mathrm{p}$. Ioo de lipides du régime sont relativement négligeables.

La formule utilisée est la suivante :

$$
\frac{\text { MS non protéique ingérée }- \text { MS non protéique excrétée }}{M S \text { non protéique ingérée }} \times \text { Ioo }=
$$

Les résultats obtenus sont consignés dans le tableau 6 .

Chez les $\mathrm{P}$, il apparaitrait d'après ces données, un abaissement du CUD des " substances non azotées " d'environ 6 points, soit $9 \mathrm{p}$. Ioo environ, valeur beaucoup plus faible que celle des protéines.

\section{DISCUSSION}

Contrairement à ce que nous attendions, il apparaît une diminution de la prise de nourriture de nos lapins privés de digestion pancréatique.

Cet abaissement de la prise de nourriture est temporaire (jusqu'à la $6^{e}$ semaine) et, en fait, relativement peu important si l'on considère qu'il faut attendre la $4^{e}$ semaine chez les $T$ pour retrouver une consommation normale. De plus, par la suite, ces 
animaux $\mathrm{P}$ reprennent une consommation alimentaire sensiblement de l'ordre de celles données par BAcQUEs et al. (I970) et PRUD'HON (I967). Toutefois, nous pensons que l'absence de suc pancréatique modifie effectivement l'appétit du lapin pour une certaine période, mais nous n'avons pas d'explication satisfaisante pour justifier ce phénomène. Nous nous contenterons de rappeler qu'il existe au niveau duodénal des substances susceptibles de jouer un rôle dans les processus d'inhibition au niveau de l'appétit (BUENo et RUCKEBUSCH I975; UGOLEV, I975). La ligature du canal pancréatique entraînerait-elle une stimulation de ces substances? et par quel mécanisme?

En ce qui nous concerne, l'intérêt de ces observations tient au fait que nous devons en tenir compte pour toute modification de croissance de nos animaux $P$.

L'étude sur l'évolution pondérale de nos lapins $\mathbf{P}$ montre que l'absence de digestion pancréatique semble modifier la croissance de ces animaux, momentanément, et plus précisément dans les 5 à 6 semaines qui suivent l'opération.

Nous pouvons donc penser que, pour une part, ce ralentissement de croissance est en relation avec une diminution de la prise de nourriture. Ce qui nous semble intéressant de noter c'est que par la suite il apparaît que la croissance reprend normalement, sans modification de régime. Toutefois, au terme des Io semaines expérimentales, les animaux $\mathbf{P}$ n'ont pas récupéré le poids des animaux $\mathrm{T}$. (Mais, nous devons signaler que nous avons gardé des animaux $\mathrm{P}$ pendant 6 mois et qu'il n'apparaît pas, à cette date, de différence globale pondérale avec les $\mathrm{T}$ ).

Tout se passe comme si à la suite de perturbations consécutives à l'absence de digestion pancréatique, il s'installait des mécanismes qui rétablissent, du point de vue appétit et croissance, un fonctionnement quasi normal à partir de la $6^{\mathrm{e}}$ semaine postopératoire. Nous devons écarter tout de suite l'hypothèse selon laquelle il pourrait y avoir régénération pancréatique (CATALA, 1972).

En fait, du point de vue croissance, le Lapin semble moins affecté par la ligature du canal pancréatique que certaines espèces. Ces troubles se manifestent de façon plus intense chez le Poulet (LEPkowsky et al, I964), le Rat (Clowes et McPherson, I964) le Chien (Svecova, I964) et s'il n'est pas signalé de modifications importantes de la croissance, c'est que le régime de ces animaux a été modifié en conséquence. BAcques et al. (I970) qui ont réalisé des ligatures du canal pancréatique chez le Lapin, ne signalent pas de variation pondérale chez ces animaux.

L'étude du CUD azoté des animaux T avant et 4 semaines après l'opération fictive, montre qu'il n'y a pas d'évolution spontanée importante de ce $\mathrm{CUD}_{\boldsymbol{a}}$ chez les animaux adultes, dont l'âge passe de $I_{4}$ et I 5 semaines à I 8 et I 9 semaines. Nos valeurs se situent proches de celles de PROTo (Ig68). Par contre, ces résultats sont différents de ceux observés, chez des lapins en croissance, dont la chute du CUD a été évaluée à ro points entre la $6^{\mathrm{e}}$ et $9^{\mathrm{e}}$ semaine (LEBAS, I973).

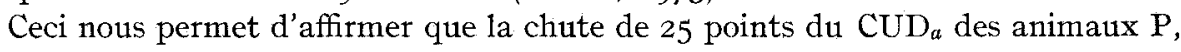
4 semaines après l'opération, représente une diminution de la digestion protéique d'environ $30 \mathrm{p}$. Ioo. Chez le Lapin, la digestion pancréatique semble donc avoir un rôle relativement important par rapport aux autres espèces. Dans les mêmes conditions, la ligature du canal pancréatique chez le Porc (ANDERSON et AsH, I97I), le Chien (Svecova, I964) et URAm et al. (I960) chez le Rat, ne modifie que peu la digestibilité des protéines alors que celle des glucides et des lipides est fortement affectée.

Cependant, ici encore, il est difficile de comparer nos résultats avec ceux de certains de ces auteurs qui modifient les régimes des animaux. C'est ainsi qu'URAM 
signale que, chez des rats privés de sécrétion pancréatique, les variations du CUD $a$ sont différentes suivant que ces animaux reçoivent un régime à base de protéines végétales ou de protéines animales. Nous pensons que, chez nos animaux, nourris aux aliments concentrés du commerce ( $\mathrm{I} 6 \mathrm{p}$. Ioo de protéines), les enzymes pancréatiques doivent jouer un rôle important (CORRING et al., 1972). Nous pouvons émettre une hypothèse selon laquelle des animaux nourris à la luzerne et à l'avoine, pourraient avoir une perturbation de la digestibilité protéique moins importante après ligature du canal pancréatique (travaux en cours de vérification).

Bien que nous ayons situé notre expérimentation au moment où la croissance est la plus affectée, il est important de noter que, malgré un tel abaissement de la digestibilité protéique, le Lapin peut encore assurer une certaine croissance. On est également en mesure de se demander quelle serait la variation du $\mathrm{CUD}_{a}$ dans la période où la croissance semble la plus proche de la normale?

Il faut donc admettre qu'il existe d'autres mécanismes susceptibles d'assurer une digestion efficace pour l'hôte, soit au niveau des enzymes intestinaux, soit au niveau des microorganismes. Or, si le rôle des microorganismes dans les processus de digestion reste à définir, il suffit de rappeler l'importance de leur métabolisme au niveau cæcal (MARTY, I97I). De plus, nous avons pu établir, il est vrai théoriquement, mais nous sommes en train de le vérifier expérimentalement, que le CUD des glucides est très faiblement affecté (de l'ordre de $9 \mathrm{p}$. roo) chez les $P$. Nous savons quel rôle important les microorganismes jouent dans la digestion des glucides et plus particulièrement chez le Lapin (Cools et Jeuniaux, I96I) (Yoshida et al., I968) (Tamm, I974).

Une deuxième hypothèse selon laquelle le rôle des microorganismes serait prépondérant dans les processus de digestion chez nos animaux à canal $\mathrm{P}$, repose sur nos travaux précédents. Dans ce travail, nous avons observé (CATALA et BonNAFous, 1974) une élévation de $30 \mathrm{p}$. Ioo des microorganismes tout le long du tube digestif chez les $\mathbf{P}$ et, parallèlement, une exaltation de 1'activité du facteur lytique au niveau colique. Ces résultats seraient en faveur de l'hypothèse selon laquelle il pourrait y avoir une récupération azotée compensatrice dans le côlon de ces animaux $\mathrm{P}$ en réponse à leur déficience pancréatique.

\section{CONCLUSION}

La ligature du canal pancréatique chez le Lapin entraîne une diminution de la prise de nourriture jusque vers la $6^{\mathrm{e}}$ semaine post-opératoire. Il apparaît, parallèlement à cette période, un ralentissement de croissance qui se traduit par une diminution de gain de poids qui n'atteint jamais plus de to p. roo, alors que l'abaissement du $\mathrm{CUD}_{a}$ est de l'ordre de $30 \mathrm{p}$. Ioo. Au-delà de cette période, l'évolution pondérale de ces animatx semble reprendre normalement.

Dans nos conditions expérimentales, il semble donc que, chez le Lapin, la participation pancréatique dans les processus de digestion, soit importante. Mais, nous pensons qu'il existe, chez ces animaux, des mécanismes de remplacements susceptibles d'assurer, en cas de déficience pancréatique, une croissance quasi normale. Nous avons tout lieu de croire que, pour une grande part, ce sont les bactéries qui jouent ce rôle, principalement dans les parties postérieures du tube digestif. 
SUMMARY

\section{VARIATIONS IN FEEDING BEHAVIOR, GROWTH AND DIGESTIBILITY IN RABBITS WITH A LIGATED PANCREATIC DUCT}

The role of the pancreas in digestion is studied in 30 rabbits. Feeding behavior, growth and nitrogen conversion ratio are observed after ligature of the pancreatic duct. Rabbits are fed a dry commercial feed containing 16 p. Ioo protein.

Feed intake and growth in $\mathrm{I} 6$ rabbits are studied in the first experiment lasting ro weeks : 8 controls $\mathrm{T}$ and 8 with the pancreatic duct ligated $\mathrm{P}$. Intake decreases until about 4 weeks after the operation in the controls, and until 6 weeks in the operated animals. The latter also grow slower during this period, but afterwards normal ponderal growth resumes.

Nitrogen conversion ratio is studied in a second experiment on 2 lots of 7 controls and operated subjects ; protein digestive decreases by about $30 \mathrm{p}$. I00, 4 weeks after the duct is ligated.

\section{RÉFÉRENCES BIBLIOGRAPHIQUES}

Andersos D. M., Asm R. W., 1971. The effect of ligating the pancreatic duct on digestion in the Pig. Proc. Nutr. Soc., 30, I-34 A.

Bacoues C., Demigne C., Vaiton C., i97o. Influence de la ligature du canal pancréatique et de la cæcumectomie sur l'excrétion fécale des lipides chez le lapin. C. R. Soc. Biol., Fr., 164, I500-I504.

Baxter S. G., I931. Continuous pancreatic secretion in the Rabbit. Amer, J. Physiol., 96, 343.

Bonnafous R., Raynaud P., rg68. Mise en évidence d'une activité lysante du côlon proximal sur les micro-organismes du tube digestif du Lapin. Arch. Sc. Physiol., Fr., 22, 57-64.

Bueno L., Ruckebusch Y., r974. Contrôle de l'ingestion alimentaire par les chémorécepteurs duodénaux chez les Ruminants. C. $R$. Acad. Sc., D, 279, 409-4I 2 .

CATAlA J., 1972. Quelques modifications nutritionnelles et digestives consécutives à la suppresion de la sécrétion pancréatique exocrine chez le Lapin. Thèse $3^{\mathrm{e}}$ cycle, Toulouse, Université Paul Sabatier.

Catala J., Bonnafous R., r974. Variations quantitatives de la microflore intestinale du Lapin, après ligature du canal pancréatique. C. R. Acad. Sc. Paris, 278, 335 I-3353.

Clowes G. H. A. Jr., McPherson L. B., 195I. Production of fatty livers by ligature of the pancreatic duct in Rats. Amer. J. Physiol, 165, 628-638.

Cools A., Jecriaux C., r96r. Fermentation de la cellulose et absorption des acides gras volatils au niveau du cæcum. Arch. Intern. Physiol. Biochem., Belg., 69, I-3.

Corring T., Lebas F., Courtot D., I972. Contrôle de l'évolution de l'équipement enzymatique du pancréas exocrine du Lapin de la naissance à 6 semaines. Ann. Biol. anim. Bioch. Biophys., $1222 \mathrm{I}-23 \mathrm{I}$.

Demigne C., Vaiton Ch., Bacgues C., 1974. Le rôle de la sécrétion pancréatique exocrine dans l'absorption intestinale du cholestérol chez le Lapin. Ann. Biol. anim. Bioch. Biophys., 14, 499-519.

LeBAS F., I969. L'alimentation du Lapin. L'alimentation et la vie, 57, 245-268.

LEBAS F., I973. Variations chez le Lapin des coefficients d'utilisation djgestive de la matière sèche, de la matière organique et de l'azote en fonction de l'âge, de la race et du sexe (abstr.). Ann. Biol. anim. Bioch. Biophys., 13, 767 .

Lepkowsky S., Nalbandov A. V., Dimick M. K., I964. Growth and reproduction of depancreatized Chickens. Endocrinology, 74, 207-221.

MARTY J., I97I. Origine et intérêt nutritionnel des acides organiques et des acides aminés dans le caecum du Lapin. Thèse Doctorat Sciences Naturelles, Université Paul Sabatier, Toulouse.

Pekas J. C., Hays V. W., Thompson A. M., I964. Exclusion of the exocrine pancreatic : secretion effect of digestibility of soyabean and milk protein by baby Pigs at various ages. J. Nutr., U.S.A., 82, 277-286.

Рвото $r$., г964. Studio preliminare nella variabilita della sigeribilita nel Coniglio. Prod, anim., 3, $33 \mathbf{I}-335$.

Prud'hon M.; I967. L'appétit du Lapin alimenté à sec. Bull. tech. Inf. Ingrs. Serv. Agric., 219, I-I6.

Rothman S. S., r964. Exocrine secretion from the isolated Rabbit pancreas. Nature, 204, 84-85.

SvECOYA O. I., r964. Digestion of nutrients in Dogs with pancreatic secretion excluded on different types of feeding. lop. Pitan., 28, $\mathrm{n}^{\circ} 3,58-6 \mathrm{r}$.

TамM Ch., 1974. Transformation of organic compounds by microbial enzymes. Febs. letters, 48, 7-21. 
I'iscornia
$267-271$. O. M., Dreiling D. A., I966. Does the pancreatic gland regenerate. Gastroenterology, 51, Ugolev A. M., 1975. The existence of a special appetite regulating intestinal hormone, Arenterine. Proc. Acad. Sci., U.R.S.S., 4, 64-66.

URAM J. A., Friedman L., KLINE O. L., I 960 . Relation of pancreatic exicrine to nutrition of the Rat. Amer. J. Physiol., 199, 387-394. Vrallard V., Raynaud P., I968. Présence d'un agent bactériolytique dans le contenu de l'estomac
du Lapin. J. Physiol., 60, 323.

Yoshida T., Pleasants J. R., Reddy B. S., Wostmann B. S., 1968. Efficiency of digestion in germ-free and conventional Rabbits. Brit. J. Nutr., 22, 723-737. 